\title{
Blockchain and its Application in Non-Fungible Tokens
}

Vishwas Magotra" ${ }^{1}$, Kamble Prithviraj ${ }^{1}$, Saharsh Patel ${ }^{1}$, Priyanka Gupta ${ }^{2}$

${ }^{*}$ Computer Science and Engineering, Lovely Professional University, Phagwara, Punjab, India

${ }^{2}$ School of Computer Science and Engineering, Lovely Professional University, Phagwara, Punjab, India

\begin{abstract}
Article Info

Volume 7, Issue 2

Page Number: 631-633

Publication Issue :

March-April-2021

Article History

Accepted : 25 April 2021

Published : 30 April 2021

Blockchain has always been considered as the solution to almost every problem that our world experiences in any form of transaction, one of its booming applications is non-fungible tokens. With increasing use of cryptocurrencies, and new assets that one can buy using it, non-fungible tokens are in the buzz, and through this journal we are trying to help people and upcoming generations understand non-fungible tokens and the variety of areas in which it can be used. Keywords : Blockchain, Non-Fungible Tokens, Smart Contracts, Cryptocurrencies
\end{abstract}

\section{INTRODUCTION}

In today's world where trust and money do not go hand in hand because of the crashing economies and losses that people go through, all kind of assets fall prey to frauds, not getting paid, not having a system to track royalties, ownership etc. Blockchain hence provides solution to these problems.

\section{Literature Review}

This section reviews the literature used in this paper. Blockchains are public registers such that all transactions are accumulated in the list of blocks[1]. This technology uses a simple concept of chain of blocks linked with each other, where every is block having the address of the next block and some data such as timestamp of when that block was created.
Non-fungible on the other hand means something that is unique and cannot be replaced. For example: 1 Bitcoin is sold in return of 1 bitcoin, there is not difference but that is not true in the case of nonfungible tokens[2].

A non-fungible token (NFT) is a unit of data stored on a digital ledger, called a blockchain, that certifies a digital asset to be unique and therefore not interchangeable[3].

NFTs can be used to transact items/files such as photos, videos, audio, and other types of digital files. Accessing the original file, is not however restricted to the buyer itself. While copies of these assets are available for anyone to access, NFTs are stored on blockchains to give the owner a proof of ownership which is different from copyright. 


\section{III.Working of Blockchain}

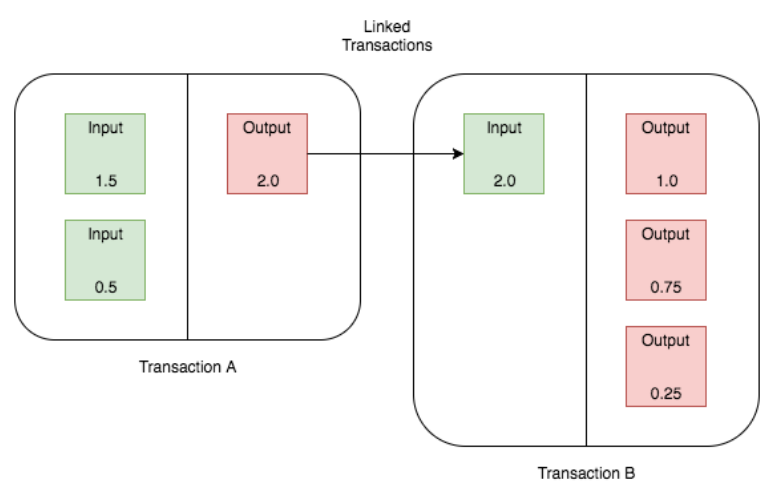

The first block of a blockchain is called genesis block Fig. 1: An example of two blocks in a blockchain. This block does not have any parent block[1].

Block also has its own constituent parts which includes block header, block version, Merkle tree root hash, timestamp, nBits, nonce, parent block hash. In addition to these components the block also contains a transaction counter and transactions.

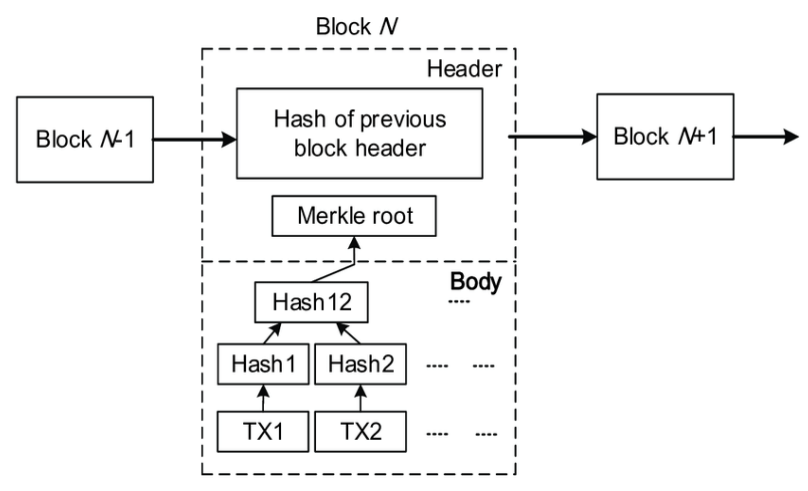

Fig. 2 : Structure of a block in blockchain

Block Version: It tells about the particular version the block is using

Merkle Tree: It is a tree of hashes of different blocks in the chain

Timestamp: Tells about the exact time at which the block was mined

Parent Block Hash: It the hash of the previous block

\section{Potential Applications based on Blockchain}

\section{(i) Implementation of Blockchain in Healthcare}

Managing medical records and patient's data, improvement in the health and other data that can be used to detect patterns in getting healthier.

(ii) Implementation of Blockchain in Banks

Blockchain can be used in making a centralized system to help banks serve their clients with high security and privacy[4].

(iii) Implementation of Blockchain in Real Estate Blockchain can be efficiently used to make real estate business hassle-free. Using smart contract, one can easily buy and sell physical assets using cryptocurrencies.

\section{Use of Non-Fungible Tokens}

Most NFTs are bought/sold on Ethereum blockchain. There are marketplaces for buying and selling ownership of these digital asset. Ethereum is a cryptocurrency just like other cryptocurrencies some of which are bitcoin, IOTA.

NFTs are one of the biggest assets in which people are investing. It will be more like a social status in the upcoming years, just like how brands are.

Owner: Anyone who can create artistic asset or owns an asset and is willing to share or sell is an owner. The owner can sell the asset by creating an NFT of it. One can create an NFT using various NFT marketplace available by simply uploading the asset to the marketplace and assigning a price to sell.

Buyers: Buyers are basically anyone who wishes to buy NFTs. People collect digital cards, gifs, images etc. anything that can be converted to NFT.

Collectibles: There are series of NFTs that can be bought together and hence have relation amongst them. 
NFTs can also be context-centric, for example character skins in a video game, objects in a virtual game etc.

\section{Smart Contract}

These are predetermined set of rules that help make decision in transactions. Basically, a program that listens to events and responds accordingly to the event. For example: using blockchain in selling/buying land. Buyer sends the money and seller send the documents of the land and once the transactions is verified, the smart contract is coded such a way that on verification, the money is transferred to the seller's account and documents are transferred to the buyer.

\section{Consensus}

In blockchain, consensus is used to figure out the untrusted nodes[5]. This helps to identify the blocks that have been changed or modified. The longest chain is the one that is considered the original one.

\section{Properties of Blockchain}

a. Security: Blockchain uses cryptography algorithms to convert data to hash value that can only be decrypted by the user's private key.

b. Transparency and Integrity: In blockchain, everybody has a public key as well as a private key, and these keys are used for encryption and decryption which ensures transparency and integrity on blockchain.

c. Immutable Storage: Blockchain has immutable storage, meaning once something is in the blockchain, it cannot be altered, and if something is altered, the hash value changes, hence whole chain will become invalid.

\section{Mining and Miners}

The process of adding blocks to the blockchain is called mining. This process requires recourses like computational power etc. and those who provide such resources are called miners. Miners take transactions form the mining pool and validate it by checking for faults and errors in the chain.

\section{Conclusion}

The speed at which technology is changing today, makes it difficult to predict the future but one thing is clear that blockchain is the future and will be the core technology in near future for almost every field and will eventually evolve to a new form to help and serve humans.

\section{REFERENCES}

[1]. Zibin Zheng et al, An Overview of Blockchain Technology: Architecture, Consensus, and Future Trends, IEEE International Congress on Big Data (Big Data Congress), 2017

[2]. https://en.wikipedia.org/wiki/Non-fungible_token

[3]. Dean, Sam(2021-03-11) “\$69 million of digital art? The NFT craze, explained" Los Angeles Times.

[4]. Sumit Soni et al, A Comprehensive survey on Blockchain: Working, security analysis, privacy threats and potential applications.

[5]. L. Lamport, R. Shostak, and M. Pease, "The byzantine generals problem," ACM transactions on Programing Languages and Systems

\section{Cite this article as :}

Vishwas Magotra, Kamble Prithviraj, Saharsh Patel, Priyanka Gupta, "Blockchain and its Application in NonFungible Tokens", International Journal of Scientific Research in Computer Science, Engineering and Information Technology (IJSRCSEIT), ISSN : 2456-3307, Volume 7 Issue 2, pp. 631-633, March-April 2021. Available at doi : https://doi.org/10.32628/CSEIT2172135 Journal URL : https://ijsrcseit.com/CSEIT2172135 